\title{
Automatic Lumbar tracking based on image processing and particle filter
}

\author{
Yuchen Liu, Yingdong Ma (Corresponding Author), Zhihao Du \\ College of Computer Science \\ Inner Mongolia University \\ Hohhot, China \\ 359281963@qq.com, csmyd@imu.edu.cn, yogine@163.com
}

\begin{abstract}
Lumbar motion is one of the most widely used information to diagnose low back disorder. By using the Digitalized Video Fluoroscopy (DVF) technique, a sequence of $\mathrm{X}$-ray frames can be acquired, which shows the movement of patient vertebra. But the $\mathrm{X}$-ray frame sequence is usually indistinct and having extra information. These interferences may lead to problems when doctors analyze the lumbar motion. In this paper, we propose an image histogram analysis based approach to deal with interferences in the $X$-ray video sequence. The method also employs a particle filter to implement automatic detecting and tracking of lumbar motion.
\end{abstract}

Keywords-component;lumbar motion; digitalized video fluoroscopy; histogram analysis; particle filter; automatic tracking

\section{INTRODUCTION}

Nowadays, low back disorder is becoming the most popular disease among people. Usually, doctors diagnose the disease by using the X-ray film. However, the main problem of X-ray films is that the image quality is poor due to the limitation of image acquisition devices. As this reason, manually disease diagnosis is often suffering inaccurate and more seriously, may lead to misdiagnosis.

Some computer vision based approaches have been proposed to help doctors diagnosing lumbar disease and achieved some results. But there are some drawbacks in these approaches, for example: inadequate utilization of available information and requirement of manually marking the position of lumbar bones at the first frame of the video sequence. In this work, we cope with these problems using a method that combines histogram analysis and a particle filter. The remainder of this paper is structured as follows. Section 2 introduces related work on medical image processing and lumbar tracking. Section 3 explains methods of image processing to analyze and improve frames of video sequence and the particle filter based tracking approach. Section 4 shows the experiment results. And finally, we conclude the work in Section 5.

\section{RELATED WORK}

Some methods have been developed for lumbar bones detection and tracking. The typical approach is based on setting control points manually to obtain contours of all lumbar bones [4][8][12]. This approach suffers from inaccurate landmark position given by system users and indistinct images. These problems may lead to shift in object tracking. Some approaches use medical image data to build template of lumbar bone and implement tracking by using template matching [1][6][10]. The main benefit of these approaches is accurate than other methods. But drawbacks of these approaches are also obvious. Modeling template is time consuming and need large training data. The template is fixed after modeling which will make it cannot fit different lumbar bones due to different position and view point. Some approaches cannot eliminate impact of geometric distortions caused by medical image acquisition device [2][3][7]. Some approaches use active contour method [9][11]. But active contour method is sensitive to image quality which can be impacted by complex environment or change of illuminate.

\section{Automatic LUmbar SEgmentation AND TRACKING}

\section{A. Image Processing and Segmentation on Lumbar Motion Video Frequency}

By analyzing the video sequence (Figure 1), some problems of X-ray film can be found. These problems are the main resources of interference for lumbar detecting and tracking:

- The original video frames show image parameters of medical image acquisition devices. However, this information has no relationship to the video targets and disturbs automatic lumbar segmentation and tracking.

- The video frames contain lumbar, other body organs, part of the image acquisition devices, and some background information, such as the device parameters. It makes the gray level range of original X-ray films is very large. Some nearby body organs have similar pixel gray value to the lumbar.

- $\quad$ The original X-ray films are indistinct. It is difficult to distinguish the edge and contour of lumbar from other body organs. 


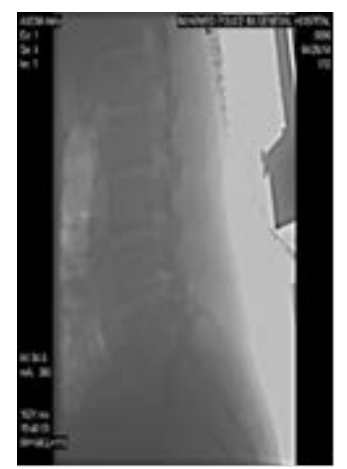

Figure 1. Original X-ray film image, collected by medical image acquisition devices.

To deal with problems of the original X-ray film image, three main steps have been applied, including image histogram equalization, image gray level adjustment and image cutting.

Image histogram equalization is a method which is commonly used to Image enhancement in image processing field. The main idea of histogram equalization is briefly described as fellow.

For a discrete grayscale image $x, n_{i}$ be the number of appearance frequency of gray level $i$. Therefore, probability of occurrence of a pixel of the gray level $i$ in the image is

$$
p_{x}(i)=\frac{n_{i}}{n}, i \in 0, \ldots, L-1,
$$

where $L$ is the total number of gray levels of the image (usually 256 for grayscale image), $n$ is the number of pixels in the image.

We can also define the cumulative distribution function (CDF) corresponding to $p_{x}$ as

$$
c d f_{x}=\sum_{j=0}^{i} p_{x}(j) .
$$

In this approach, we create a transformation of the form $y=T(x)$ to produce a new image which with a flat histogram. Using the properties of the CDF, we can define a map between the original pixel value $y$ and the transformed pixel value $y$ ' as:

$$
c d f_{y}\left(y^{\prime}\right)=c d f_{y}(T(k))=c d f_{x}(k),
$$

where $k$ is in the range $[0, L)$. Because we use a normalized histogram of $x$, the function maps the levels into the range $[0,1]$. Thus it is necessary to map the values back into the original range of the image. The map function is

$$
y^{\prime}=y *(\max (x)-\min (x))+\min (x) .
$$

The original image sequence has very low contrast. It makes pixels of lumbar bones and nearby body organs has similar gray values. In order to enhance the contrast of original images and highlight the part of lumbar bones, we use histogram equalization as the first step to process the original images.

After this step, we acquire distinct results from the original images (Figure 2).

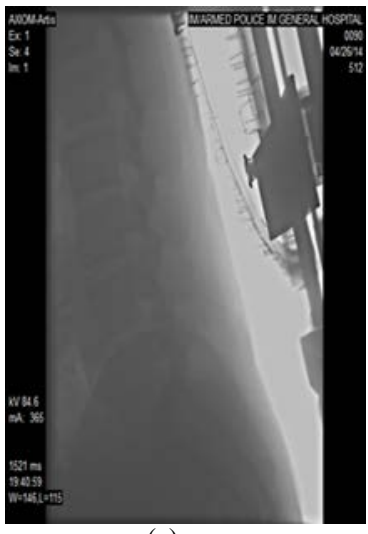

(a)

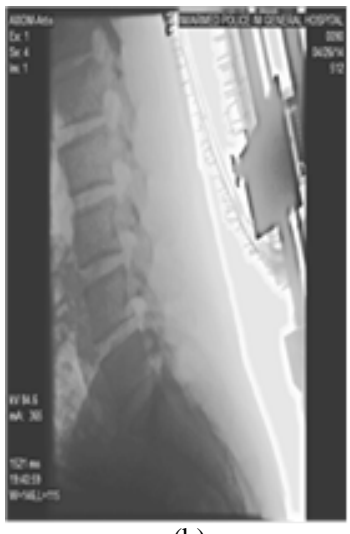

(b)
Figure 2. Original image (a) and image after histogram equalization (b)

Compare the two images, we find the image after histogram equalization is more distinct than the original image. But the contrast of other parts, with similar pixel gray value to the lumbar part, is also enhanced. These parts insist of the medical image acquisition devices at upper right corner, viscera and musculature in the image (Figure 2(b)).

After histogram equalization, clarity of images is enhanced. But interferences are also obvious. In order to further enhance the part of lumbar bones, we use gray level adjustment technique.

The gray level adjustment (or gray level transform) is a method that enhance image content which is in a certain gray level or color range by adjusting luminance of grayscale image or color matrix of color image. This method can effectively filter parts which exceed or under a certain threshold, as well as keep the completeness of source images.

In our approach, we use gray level adjustment to process the medical image acquisition devices at upper right corner, viscera and musculature in the image. As a result, images after gray level adjustment have better result: the lumbar bones are clear than before (Figure 3(b)).

Compare the images in Figure 3, image areas of the lumber bones become more distinct after processing. Moreover, most of the medical image acquisition devices, viscera and musculature are obviously eliminated.

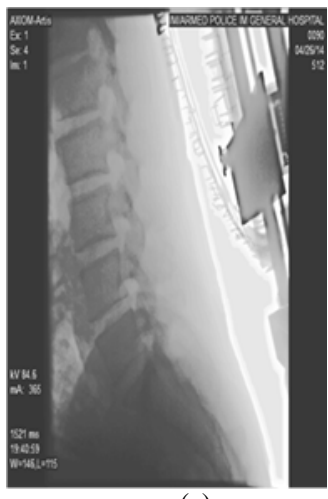

(a)

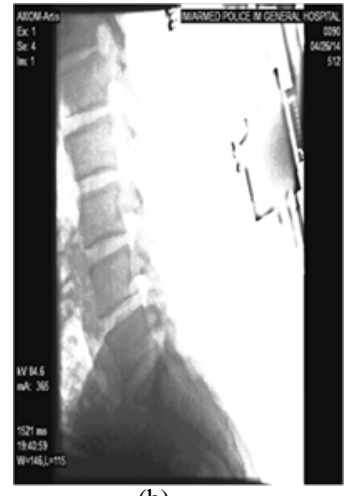

(b)
Figure 3. Image after histogram equalization (a) and image after histogram equalization and gray level adjustment (b). 
After the step of histogram equalization and gray level adjustment, the lumbar part of the images is more distinct. But existence of redundant information still have negative effect on tracking process. As the goal of this work is to track lumbar motion, in order to focus on the lumbar bones, we use image cutting to remove redundant information.

By analyzing the images, we find that pixel gray value of most parts in the images is similar to the lumbar part. If we segment the images simply using a threshold method, interfered parts may remain in the image and affect the performance of object tracking. Therefore we consider the architectural feature of the images. It can be found that lumbar bones in the images arranged approximately in the vertical direction. Thus, in the proposed method, sum of pixel gray value (SPGV) in each vertical line of the images is computed. Comparing the SPGV distribution curve in each image, there is a similar part of SPGV in the image sequence (Figure 4). As pixels of lumbar bones have smaller gray values, it is obviously that this part represents the lumbar bones. Therefore we use the SPGV in vertical direction as threshold to cutting images. As we can see from Figure 5, the lumbar bones remain in the image and most redundant and interfered parts are removed (Figure 5).

After all the processing steps, we get a more distinct and fewer interference image and, more important, the whole lumbar bones remain in the images (Figure 5(b)).

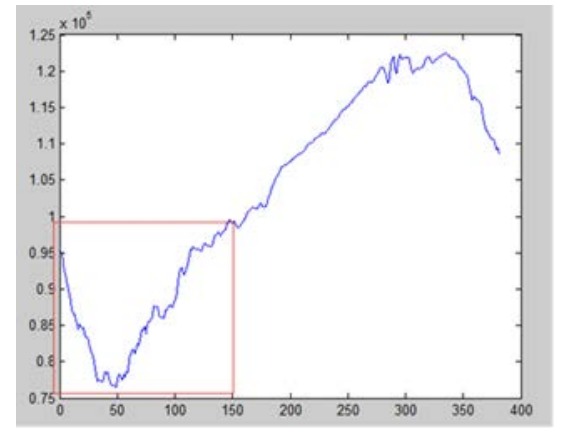

(a)

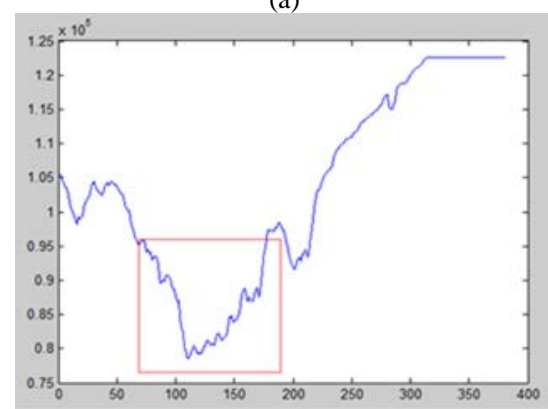

(b)

Figure 4. Sum of pixel gray value in each vertical line of the first frame (a) and 64th frame (b)

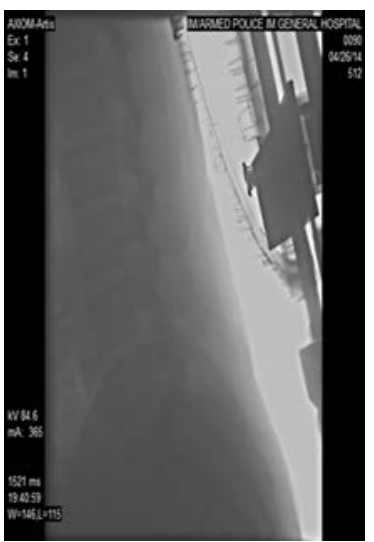

(a)

Figure 5. Original image (a) and after our processing steps (b)

\section{B. Automatic Lumbar Motion Tracking}

By observing the image sequence, we find the lumbar motion can be divided into two phase: from flexion to upright and from upright to extension. We compute the motion displacement of lumbar bones frame by frame and get characteristic of the motion. During the motion process, displacement in $\mathrm{x}$ direction is increasing, displacement in $\mathrm{y}$ direction increasing at first phase and decreasing at second phase, rotation angle of lumbar bones also increasing. Increments of displacement in $\mathrm{x}$ and $\mathrm{y}$ direction and rotation angle are almost fixed. Also, the distance between two adjacent bones is almost fixed.

According to these motion displacement, the known position $\mathrm{L}_{\mathrm{t}-1}$ of lumbar at time $\mathrm{t}-1$ can be used to compute the position $\mathrm{L}_{\mathrm{t}}$ of lumbar at time $t$. Recursive function of this procedure can be represented as

$$
L_{t}=f\left(L_{t-1}, n_{t-1}\right) .
$$

Before lumbar bones tracking, regions of interest (ROI) in the images should be located to extract characteristics of lumbar bones. Tracking is initialized by manually setting an initial ROI in the first frame and computing characteristics in this ROI region. Then, using the characteristics of the first ROI and the Bhattacharyya distance between two adjacent bones, the rest four ROI (assuming total five ROI) can be computed.

According to the above, state of lumbar motion can be described recursively. Therefore we choose particle filter as tracking approach.

Particle filter is a Monte Carlo method that uses a set of random samples with associated weights to represent required posterior density function and computes estimates based on these samples and weights.

In our approach, particle filter estimates the posterior distribution $p\left(L_{t} \mid N_{1: t}\right)$ of the unknown motion state $L_{t}$ of the lumbar motion frame by frame from the image sequence $N_{1: t}=\left\{N_{1}, \ldots, N_{t}\right\}$. From frame $t-1$ to $t$, the particle filter generates samples (or particles) according to the prior distribution of a state transition model $p\left(L_{t} \mid L_{t-1}\right)$ for each lumbar to predict the position in frame $t$. The 
observation model gives the likelihood of the fit between the contour according to the particles and the original ROI edge. The best particle is the one best matches the characteristic of the ROI. The particles are then resampled and the state estimate $\hat{L}_{t}$ is approximated from the posterior distribution $p\left(L_{t} \mid L_{t-1}\right)$ by a set of particles with associated weights. The control vector $C_{t}$ of frame $t$ is acquired from $C_{t-1}$ of frame $t-1$. Then $C_{t}, \hat{L}_{t}$ after which $L_{t}$ and $\hat{L}_{t}$ are passed back to the particle filter for the next iteration.

\section{EXPERIMENTS AND RESULTS}

\section{A. Experiments}

After we acquire the video frequency collected by the Digitalized Video Fluoroscopy system, we use the proposed method to process every frame of the image sequence. Then we composite all frames into a video sequence and use this video sequence to implement lumbar tracking.

In order to test our approach, we implement another typical segmentation and tracking method based on image smooth, histogram equalization and particle filter. The results of this method is shown in Figure 6. To compare the two approaches, we show initial state of the first frame and the 50th frame in tracking process. Tracking shift is obviously in the method based on image smooth, histogram equalization and particle filter, while performance of our method is well.

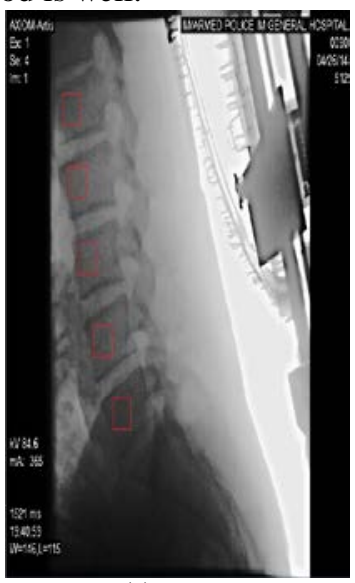

(a)

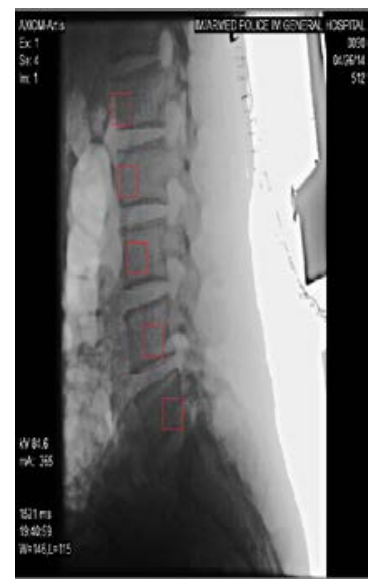

(b)

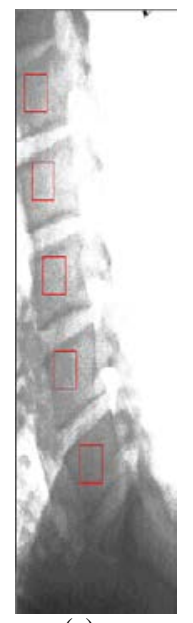

(c)

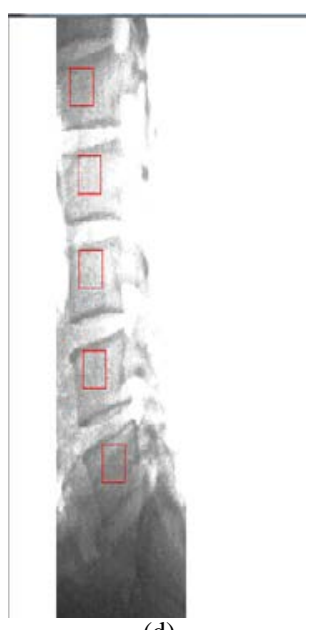

(d)
Figure 6. Comparison between tracking process based on image smooth, histogram equalization and particle filter (a), (b) and our approach (c), (d).

Compare with other approaches, our method is more distinct and more accurate. After image processing using the proposed method, the lumbar part becomes distinct and have clear edge for identification. In the proposed approach, only the initial ROI in the first frame need to be set manually. This method not only reduce workload of manual landmark, but also increase the object tracking accuracy. It can be used to help doctors diagnosing the low back disorder and reduce the probability of misdiagnosis.

\section{B. Results}

Our final tracking results are shown in Figure 7. The red rectangles is represented the lumbar we need tracking.

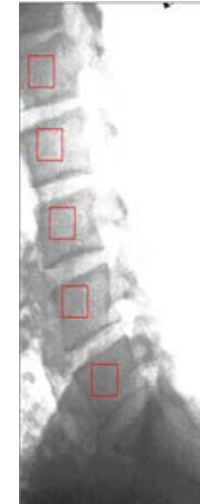

(a)

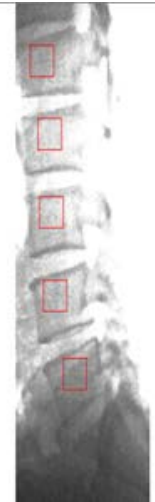

(b)

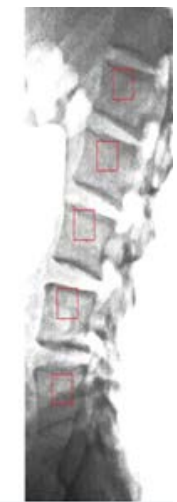

(c)
Figure 7. ROI tracking result in first frame (a), 50th frame(b) and last frame(c)

\section{CONCLUSIONS}

Our approach is suitable for general situation and reduces effort of manually control points setting. The tracking results is also accurate enough for doctors to diagnose low back disorder. The drawback of our method is time consuming. It cannot be used in real-time applications. Future works will focus on real-time implementation of the proposed method. 


\section{ACKNOWLEDGMENT}

This work is supported by National Natural Science Foundation of China (No:61461039), Program of Higherlevel talents of Inner Mongolia University (12305-125130).

\section{REFERENCES}

[1] Alexander Wong, Akshaya Mishra, Paul Fieguth, David Clausi, Nadine M. Dunk, Jack P. Callaghan, Shape-Guided Active Contour Based Segmentation and Tracking of Lumbar Vertebrae in Video Fluoroscopy Using Complex Wavelets, 30th Annual International IEEE EMBS Conference, 2008.

[2] J. Muggleton and R. Allen, "Automatic location of vertebrae in digitized videofluoroscopic images of the lumbar spine”, Med. Eng.Phys., vol. 19, no. 1, pp. 77-89, 1997.

[3] L. Penning, R. Irwan, and M. Oudkerk, Measurement of angular and linear segmental lumber spine flexion-extension motion by means of image registration, European Spine Joural, vol. 14, 2005.

[4] Mingzhi Xu, Yingyao Zhang, Xiaobo Xie, Hongyan Cui, Fuge Sui, Lifeng Zhao, Dongjun Wang, Automatic Lumbar Motion Analysis Based on Particle Filtering, International Conference on System Science and Engineering, 2012.

[5] M. Sanjeev Arulampalam, Simon Maskell, Neil Gordon, Tim Clapp,A Tutorial on Particle Filters for Online Nonlinear/Non-Gaussian Bayesian Tracking, IEEE Transactions on Signal Processing, vol.50, 2002.

[6] Poay Hoon Lim, Ulas Bagci, Omer Aras, Yan Wang, Li Bai, A Novel Spinal Vertebrae Segmentation Framework Combining Geometric Flow and Shape Prior With Level Set Method, Biomedical Imaging (ISBI), 2012.

[7] P. Bifulco, M. Cesarelli, R. Allen, M. Sansone, and M. Bracale, "Automatic recognition of vertebral landmarks in fluoroscopic sequences for analysis of intervertebral kinematics," Medical \& Biological Engineering \& Computing, vol. 39, pp. 65-75, 2001.

[8] S.C.B.Lam, R.Allen, Robust automated vertebra motion tracking from videofluoroscopy sequences, Advances in Medical, Signal and Information Processing, 2008.

[9] S. Wong, K. Wong, W. Wong, C. Leong, and D. Luk, "Tracking Lumbar Vertebrae in Digital Videofluoroscopic Video Automatically", Proc. MIAR 2004, LNCS 3150, pp. 154-162, 2004.

[10] T. Cerciello, P. Bifulco, M. Cesarelli, M. Romano and R. Allen, Automatic vertebra tracking through dynamic fluoroscopic sequence by smooth derivative template matching, Proceedings of the 9th International Conference on Information Technology and Applications in Biomedicine, ITAB 2009.

[11] W. Wong, D. Luk, C. Leong, F. Wong, and K. Wong, "Continous Dynamic Spinal Motion Analysis”, Spine, vol. 31, no. 4, pp. 414-419, 2006.

[12] Yingyao Zhang, Xiaobo Xie, Hongyan Cui, Fuge Sui, Lifeng Zhao, Dongjun Wang, Automatic Tracking Measurement System on Human Lumbar Vertebral Motion, International Conference on Virtual Environments, Human-Computer Interfaces and Measurements Systems, 2009.

[13] Yalin Zheng, Mark S. Nixon, and Robert Allen, Automated Segmentation of Lumbar Vertebrae in Digital Videofluoroscopic Images, IEEE Trans Med Imaging, vol.23, no.1, Jan 2004.

[14] Yalin Zheng, Mark S. Nixon and Robert Allen, Automatic lumbar vertebrae segmentation in fluoroscopic images via optimised concurrent hough transform, Proceedings of the 23rd Annual EMBS International Conference,2001. 